\title{
Analysis of Capillary Flow in Parallel Microchannel based Wick Structure with Circular and Non- Circular Geometries
}

Binjian $M a{ }^{*},+$

$\dagger^{\dagger}$ Department of Mechanical Engineering \& Automation, Harbin Institute of Technology

(Shenzhen), Shenzhen, Guangdong, China, 518055

\section{Supporting Information}

\section{S.1 Validation of constant contact angle assumption in the capillary rise}

While the contact angle formed between the liquid and the solid surface during motion is different from the static contact angle, such a difference is highly dependent on the flow velocity. As shown by Fries and Dreyer in their well-known article "An Analytical Solution of Capillary Rise Restrained by Gravity" 1, the effect of dynamic contact angle is found to be insignificant for capillary rise in microtube (e.g., capillary radius is $0.1 \mathrm{~mm}$ ) in which the capillary flow velocity varies from $0 \mathrm{~mm} / \mathrm{s}$ to as high as $35 \mathrm{~mm} / \mathrm{s}$. These geometric and flow conditions are very similar to the capillary wicking phenomenon explored in this study. Likewise, we can follow the same approach adopted by Fries and Dreyer to demonstrate that the effect of dynamic contact angle can be neglected in this study. The relationship between the dynamic contact angle and the flow rate can be estimated using the empirical correlation proposed by Bracke et al. ${ }^{2}$

$$
\frac{\cos \theta_{d}-\cos \theta_{s}}{\cos \theta_{s}+1}=-2 \mathrm{Ca}^{0.5}
$$

Here, $\theta_{d}$ and $\theta_{s}$ are the dynamic and static contact angle, respectively, and $\mathrm{Ca}$ is the capillary number given by

$$
\mathrm{Ca}=\frac{\mu v}{\sigma}
$$

where $\mu$ is the viscosity, $v$ is the velocity, and $\sigma$ is the surface tension. Figure $\mathrm{S} 1$ shows the change of the dynamic contact angle and capillary pressure with increasing flow velocity calculated for water and copper based on Equation 1 and 2. It can be found that the effect of dynamic contact angle on the capillary pressure only becomes significant when the flow velocity exceeds $0.03 \mathrm{~m} / \mathrm{s}$. Based on these results and following the same theoretical calculation procedures outlined in the 
manuscript, we can further obtain the capillary rate-of-rise in the microchannel that considers the effect of dynamic contact angle. Figure S2 shows the comparison of the capillary rate-of-rise curves obtained using a static contact angle and a dynamic contact angle in a circular microchannel with a diameter of $200 \mu \mathrm{m}$. It is evident to notice that there is only marginal difference between the results calculated using a static and dynamic contact angle. For microchannels with a smaller diameter, the effect of dynamic contact angle will become more insignificant because the capillary flow rate decreases faster over time. Therefore, it is reasonable to assume a constant contact angle for capillary rise in this study.

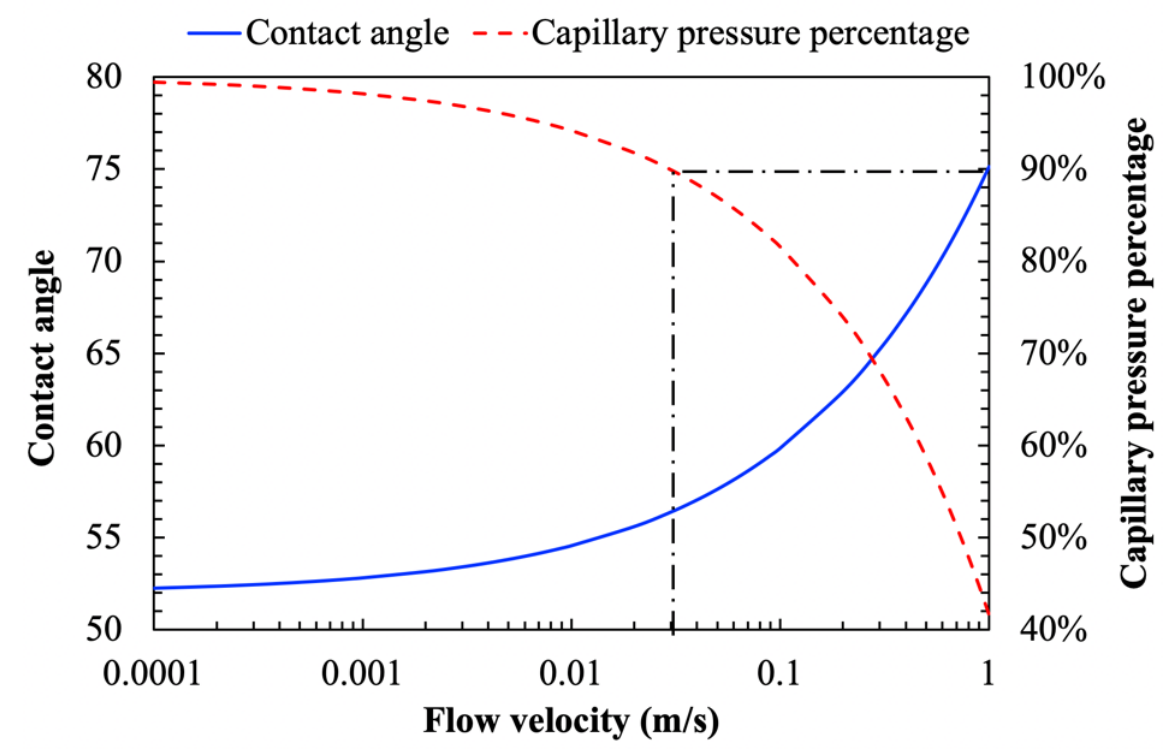

Figure S1. The variation of contact angle and capillary pressure with increasing flow velocity.

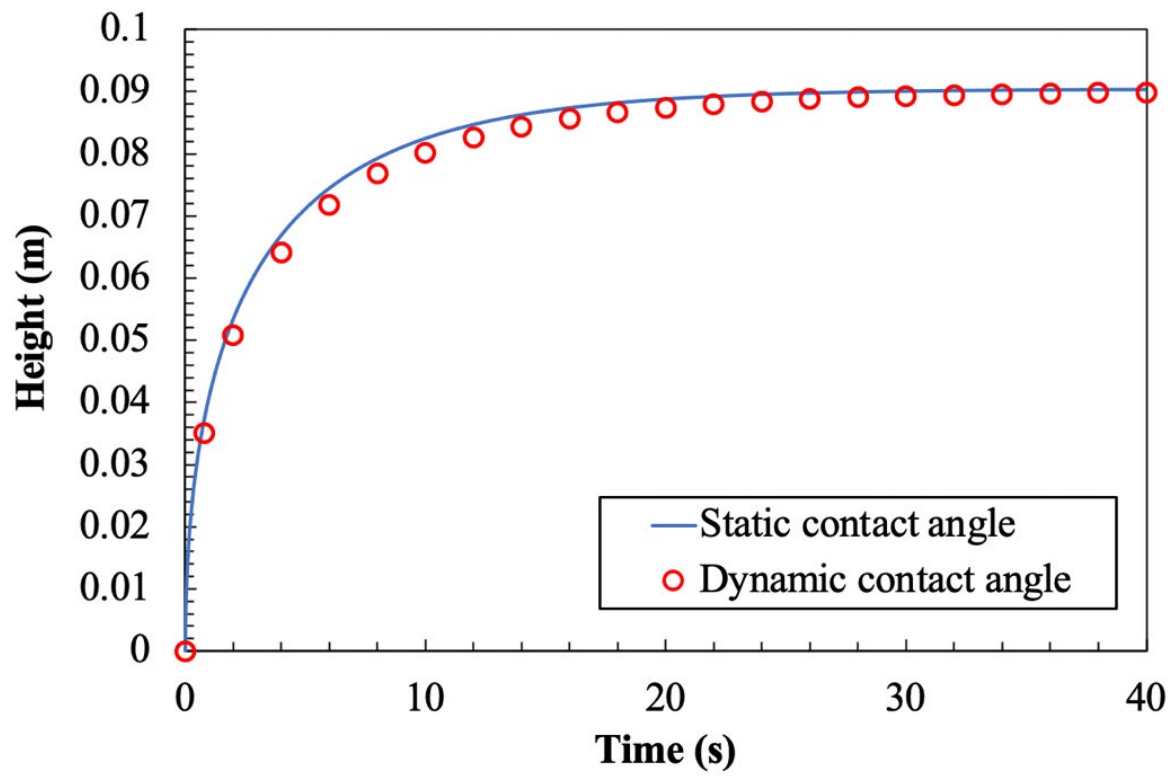

Figure S2. The comparison of the capillary rate-of-rise curves obtained using a static contact angle and a dynamic contact angle. The results are obtained for water wicking in a circular microchannel with a diameter of $200 \mu \mathrm{m}$. 


\section{S.2 Descending liquid in microchannel with an initial height beyond the capillary limit}

The theoretical limit of the capillary rise in the microchannel can be calculated based on the balance between the capillary pressure and the gravitational force, i.e.,

$$
\rho g h=\frac{4 \sigma \cos \theta}{D_{H}}
$$

where $\rho$ is the liquid density, $g$ is the gravitational acceleration, $h$ is the ultimate capillary height, $\sigma$ is the surface tension, $\theta$ is the contact angle, and $D_{H}$ is the hydraulic diameter. For the circular microchannel with a diameter of $200 \mu \mathrm{m}$ as shown in Figure 3 in the manuscript, the ultimate capillary height is calculated to be $88.7 \mathrm{~mm}$. In addition, as shown in Figure 3 itself, the capillary height almost ceases to increase completely at a capillary height of $80 \mathrm{~mm}$. Therefore, additional simulation with increasing capillary height will not yield any valuable information. Figure S3 shows the simulation results with the initial liquid height set at $h=100 \mathrm{~mm}$ which exceeds the theoretical maximum capillary height. As shown in the figure, a typical inertia oscillation behavior is observed in the initial stage during which the liquid-vapor interface is equilibrating from a flat plane to a concave curve. After $t=0.5 \mathrm{~ms}$, it is evident to notice that the liquid-vapor interface is descending during the simulation at a noticeable rate. This negative velocity indicates that the liquid is retracting from the initial location under the gravitational effect, which is consistent with the real physics.
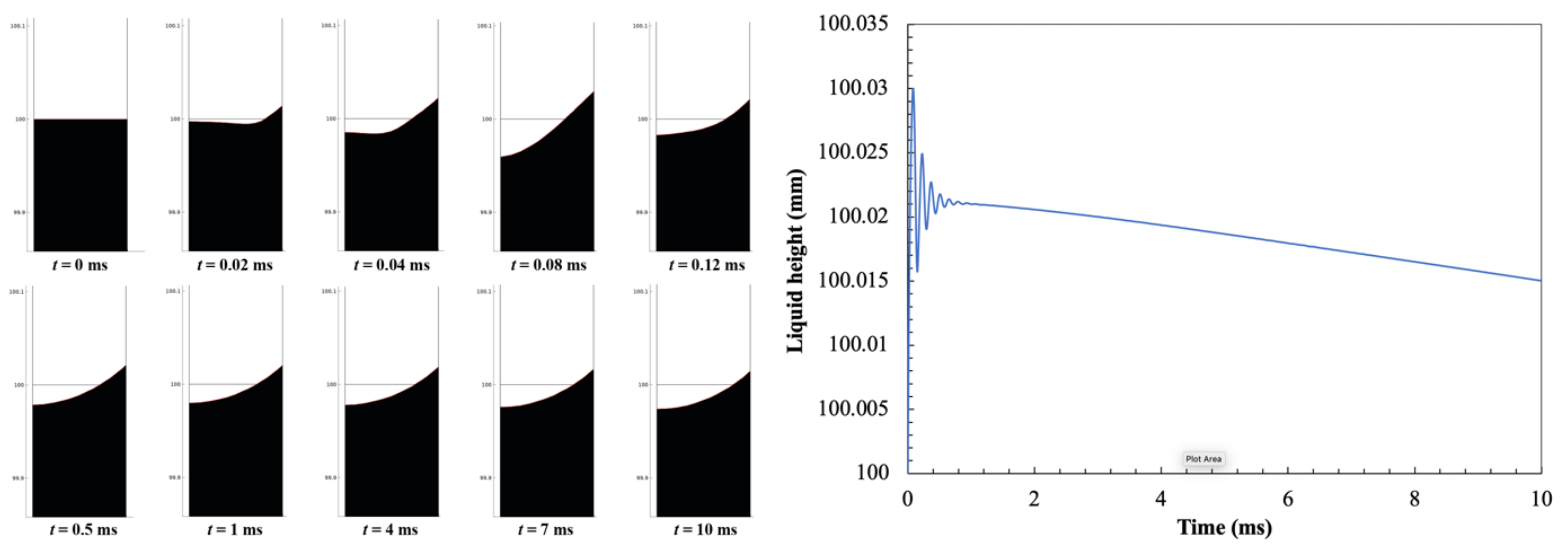

Figure S3. The simulation results of water in a circular microchannel with a diameter of $200 \mu \mathrm{m}$ and an initial height of $100 \mathrm{~mm}$. The figure on the left shows the slapshots of the liquid body taken at 10 different moments. The figure on the right shows the evolution of the contact line position over time. 
S.3 Derivation of packing density for parallel microchannels with circular and non-circular cross sections

(1) Parallel circular microchannel:

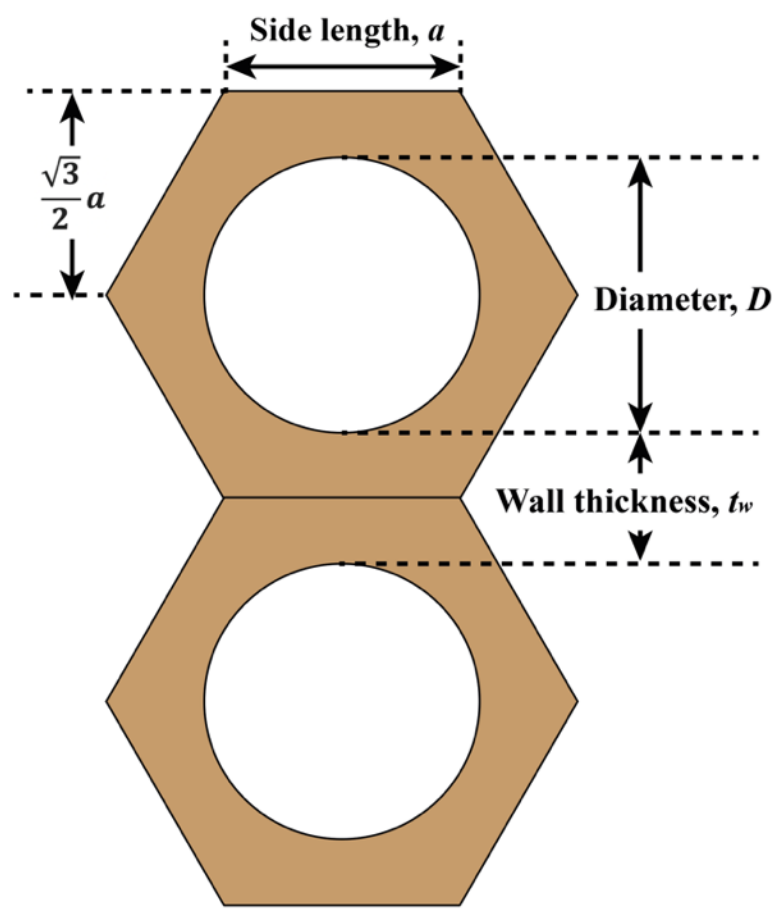

Figure S4. The schematic of parallel circular microchannels packed in a hexagonal pattern.

For parallel circular microchannel packed in hexagonal pattern, the area of the hexagon and the circle are given by

$$
\begin{gathered}
A_{\text {hex }}=\frac{3 \sqrt{3}}{2} a^{2} \\
A_{\text {cir }}=\frac{\pi}{4} D^{2}
\end{gathered}
$$

Therefore, the packing density can be calculated based on equations (4) to (9) as

$$
\psi_{\text {cir }}=\frac{A_{\text {cir }}}{A_{\text {hex }}}=\frac{\pi}{6 \sqrt{3}} \cdot \frac{D^{2}}{a^{2}}
$$

According to the geometric relationship shown in Figure S4, we have

$$
2 \cdot \frac{\sqrt{3}}{2} a=D+t_{w}
$$


Substituting equation (7) in equation (6) yields

$$
\psi_{\text {cir }}=\frac{\pi}{2 \sqrt{3}}\left(\frac{D}{D+t_{w}}\right)^{2}
$$

(2) Parallel hexagonal microchannel:

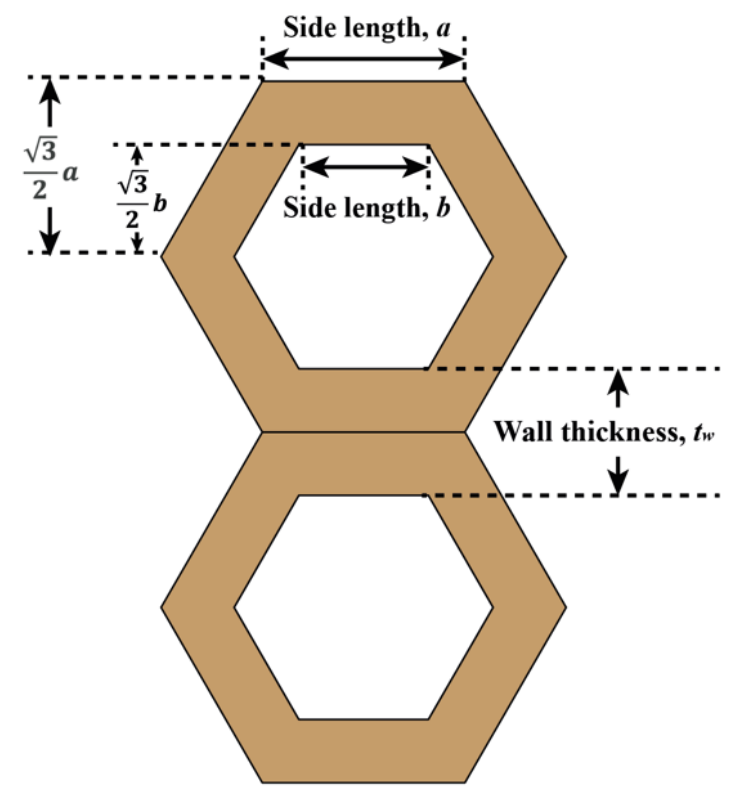

Figure S5. The schematic of parallel hexagonal microchannels packed in a hexagonal pattern.

For parallel hexagonal microchannel packed in hexagonal pattern, the packing density can be calculated by

$$
\psi_{\text {hex }}=\frac{A_{\text {inner }, \text { hex }}}{A_{\text {outer }, \text { hex }}}=\left(\frac{b}{a}\right)^{2}
$$

According to the geometric relationship shown in Figure S5, we have

$$
\frac{\sqrt{3}}{2} a-\frac{\sqrt{3}}{2} b=\frac{t_{w}}{2}
$$

Substituting equation (10) in equation (9) yields

$$
\psi_{\text {hex }}=\left(\frac{b}{b+t_{w} / \sqrt{3}}\right)^{2}=\left(1-\frac{t_{w}}{\sqrt{3} b+t_{w}}\right)^{2}
$$


For hexagonal channel, the hydraulic diameter is given by

$$
D_{H}=\frac{4 A_{\text {hex }}}{P_{\text {hex }}}=\frac{4 \cdot \frac{3 \sqrt{3}}{2} b^{2}}{6 b}=\sqrt{3} b
$$

Substituting equation (12) in equation (11) yields

$$
\psi_{\text {hex }}=\left(1-\frac{t_{w}}{D_{H}+t_{w}}\right)^{2}
$$

(3) Parallel square microchannel:

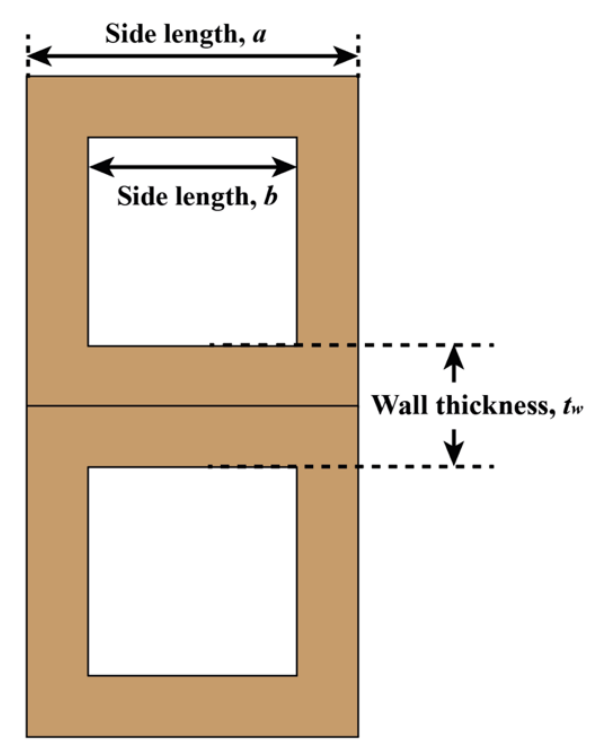

Figure S6. The schematic of parallel square microchannels packed in a square pattern.

For parallel square microchannel packed in square pattern, the packing density can be calculated by

$$
\psi_{\text {squ }}=\frac{A_{\text {inner }, \text { squ }}}{A_{\text {outer }, \text { squ }}}=\left(\frac{b}{a}\right)^{2}
$$

According to the geometric relationship shown in Figure S6, we have

$$
a-b=t_{w}
$$

Substituting equation (15) in equation (14) yields 


$$
\psi_{s q u}=\left(\frac{b}{b+t_{w}}\right)^{2}=\left(1-\frac{t_{w}}{b+t_{w}}\right)^{2}
$$

For square channel, the hydraulic diameter is given by

$$
D_{H}=\frac{4 A_{s q u}}{P_{s q u}}=\frac{4 \cdot b^{2}}{4 b}=b
$$

Substituting equation (17) in equation (16) yields

$$
\psi_{s q u}=\left(1-\frac{t_{w}}{D_{H}+t_{w}}\right)^{2}
$$

(4) Parallel triangular microchannel:

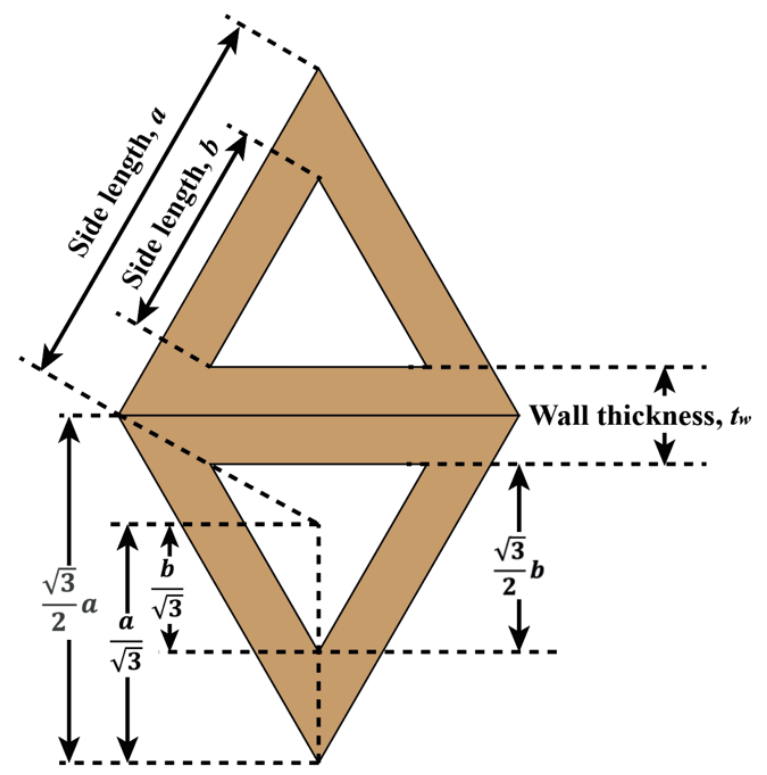

Figure S7. The schematic of parallel triangular microchannels packed in a triangular pattern.

For parallel triangular microchannel packed in triangular pattern, the packing density can be calculated by

$$
\psi_{\text {tri }}=\frac{A_{\text {inner }, \text { tri }}}{A_{\text {outer }, \text { tri }}}=\left(\frac{b}{a}\right)^{2}
$$

According to the geometric relationship shown in Figure S7, we have 


$$
\frac{\sqrt{3}}{2} a-\frac{\sqrt{3}}{2} b-\left(\frac{a}{\sqrt{3}}-\frac{b}{\sqrt{3}}\right)=\frac{t_{w}}{2}
$$

which yields

$$
a-b=\sqrt{3} t_{w}
$$

Substituting equation (21) in equation (19) yields

$$
\psi_{\text {tri }}=\left(\frac{b}{b+\sqrt{3} t_{w}}\right)^{2}=\left(1-\frac{t_{w}}{b / \sqrt{3}+t_{w}}\right)^{2}
$$

For triangular channel, the hydraulic diameter is given by

$$
D_{H}=\frac{4 A_{t r i}}{P_{t r i}}=\frac{4 \cdot \frac{\sqrt{3}}{4} b^{2}}{3 b}=\frac{b}{\sqrt{3}}
$$

Substituting equation (23) in equation (22) yields

$$
\psi_{\text {tri }}=\left(1-\frac{t_{w}}{D_{H}+t_{w}}\right)^{2}
$$

As shown by equations (13), (18), and (24), the packing density of parallel microchannel structure with a hexagonal, square, triangular cross section can be calculated using the same equation. 


\section{S.4 Modification of capillary flow rate based on the packing density}

Since the packing density of parallel microchannel structure differs for different geometric configurations, the flow velocity obtained from a single channel should be modified to correctly characterize the capillary flow rate through the parallel microchannels. Now assume that the total cross-sectional area of the bulk porous media is $A_{b u l k}$ and the cross-sectional area of a single microchannel is $A_{m c}$, then the total number of channel that can be accommodated in the bulk material is given by

$$
n_{m c}=\frac{A_{b u l k}}{A_{m c} / \psi_{m c}}
$$

where $\psi_{m c}$ is the packing density of the parallel microchannel. The volumetric flow rate in a single microchannel is given by

$$
\dot{V}_{m c}=A_{m c} v
$$

where $v$ is the capillary flow velocity. Therefore, the total volumetric flow rate through the entire porous media is given by

$$
\dot{V}_{t o t}=n_{m c} \cdot \dot{V}_{m c}=v \psi_{m c} A_{b u l k}
$$

Therefore, the mass flow rate per unit area is given by

$$
\dot{V}_{\text {unit }}=\frac{\dot{V}_{\text {tot }}}{A_{\text {bulk }}}=v \psi_{m c}
$$

As shown by equation (28), the mass flow rate per unit area of the entire porous media equals to the flow velocity multiplied with the packing density.

\section{References:}

1. Fries, N.; Dreyer, M., An analytic solution of capillary rise restrained by gravity. Journal of colloid and interface science 2008, 320 (1), 259-263.

2. Bracke, M.; De Voeght, F.; Joos, P., The kinetics of wetting: the dynamic contact angle. In Trends in Colloid and Interface Science III, Springer: 1989; pp 142-149. 